\title{
Racial Disparities in Delivery Gestational Age among Twin Pregnancies
}

\author{
Jacqueline H. Grant, MD ${ }^{1}$ Catherine J. Vladutiu, PhD ${ }^{1}$ Tracy A. Manuck, MD ${ }^{1}$ \\ ${ }^{1}$ Division of Maternal-Fetal Medicine, Department of Obstetrics and \\ Gynecology, University of North Carolina, Chapel Hill, North Carolina \\ Am J Perinatol 2017;34:1065-1071. \\ Address for correspondence Jacqueline H. Grant, MD, Division of \\ Maternal-Fetal Medicine, Department of Obstetrics and Gynecology, \\ University of North Carolina, 3010 Old Clinic Building, CB \#7516, \\ Chapel Hill, NC 27599-7516 \\ (e-mail: jacqueline_grant@med.unc.edu).
}

\begin{abstract}
Objective This study aims to estimate the association between maternal race and delivery gestational age among women with twin gestations.

Study Design Secondary analysis of a prospective, randomized control trial of $17-\alpha$ hydroxyprogesterone caproate versus placebo for preterm birth (PTB) prevention in twin gestations. Non-Hispanic (NH) black and whites were included. Demographic and antenatal characteristics were compared. The primary outcome was delivery gestational age. Secondary outcomes included a composite of major neonatal morbidity. Kaplan-Meier curves estimated survival probabilities for delivery gestational age by race. Cox proportional hazards models estimated hazard ratios (HR) and 95\% confidence intervals $(\mathrm{Cl})$.

Results A total of 535 women with twin gestations were included; 150 were NH black. $\mathrm{NH}$ blacks delivered earlier than $\mathrm{NH}$ whites $(33.6 \pm 4.8$ weeks vs. $35.1 \pm 3.5$ weeks, $p<0.001)$. Differences in delivery gestational age between $\mathrm{NH}$ blacks and whites were consistent across gestation. In adjusted analyses, NH black race (HR: 1.24, 95\% $\mathrm{Cl}: 1.02-1.51)$, prior PTB (HR: $1.59,95 \% \mathrm{Cl}: 1.15-2.19)$, and cerclage (HR: $3.90,95 \%$ $\mathrm{Cl}$ : 2.00-7.60) were associated with an increased risk of earlier delivery. Major

\section{Keywords}

- preterm birth

- health disparities

- twins neonatal morbidity was higher for $\mathrm{NH}$ blacks compared with $\mathrm{NH}$ whites (12.7 vs. $7.0 \%, p=0.036)$.

Conclusion NH blacks with twin gestations have an increased risk of early delivery and neonatal morbidity compared with $\mathrm{NH}$ whites.
\end{abstract}

Preterm birth (PTB) is the leading cause of infant morbidity and mortality among nonanomalous neonates in the United States $^{1}$ and survivors face increased short- and long-term morbidities that may result in a lifetime of medical disability. $^{2}$ In adulthood, survivors are at an increased risk for adult-onset chronic metabolic, cardiovascular, and renal diseases compared with their term counterparts. ${ }^{3-5}$ The burden of PTB extends well beyond individuals and families. The Institute of Medicine estimated that the annual societal cost of PTB in the United States was at least $\$ 26.5$ billion in $2005 .{ }^{6}$
After reaching an unacceptably high rate in 2007, the PTB rate met a modest decline from 2007 to 2014, followed by a concerning increase between 2014 and 2015. ${ }^{7}$ Though the overall rate of PTB in 2015 was 9.6\% in the United States, the rate for non-Hispanic blacks was $50 \%$ higher than nonHispanic whites (13.4\%). ${ }^{8}$ Unfortunately, the reason for this disparity remains unclear, as the etiologies underlying PTB are numerous and complex and a precise cause of PTB is often difficult to ascertain. ${ }^{9}$ Differences in socioeconomic status, risky behaviors during pregnancy, and access to prenatal care may explain some of the disparity in birth
Copyright @ 2017 by Thieme Medical Publishers, Inc., 333 Seventh Avenue, New York, NY 10001, USA. Tel: +1(212) 584-4662.
DOI https://doi.org/ 10.1055/s-0037-1603764. ISSN $0735-1631$. 
outcomes between black women and those of other races. ${ }^{10}$ However, PTB disparities often persist after controlling for these factors. $^{11-14}$

In 2015 , the incidence of twin birth was 33.5 per 1,000 total births and PTB was 7.5 times more common in twins than singletons. ${ }^{8}$ Though race and plurality are significant independent risk factors for PTB, as well as other adverse perinatal outcomes, little is known about racial differences in delivery gestational age among twin gestations. The objective of this study was to assess the association between maternal black race and gestational age at delivery among a large cohort of women with twin gestations. We also sought to evaluate whether the frequency of adverse pregnancy and neonatal outcomes varies between non-Hispanic black and white women.

\section{Materials and Methods}

This is a secondary analysis of women with uncomplicated twin gestations enrolled from April 2004 to February 2006 in a multicenter, prospective double-blind randomized controlled trial of 17- $\alpha$ hydroxyprogesterone caproate (17-OHPC) versus placebo for the prevention of PTB conducted by the Eunice Kennedy Shriver National Institute of Child Health and Human Development Maternal-Fetal Medicine Units Network. ${ }^{15}$ Briefly, the trial enrolled 661 pregnant women with twin gestations and randomized them to receive either weekly 17-OHPC injections or placebo starting at $16^{0 / 7}$ to $20^{3 / 7}$ weeks gestation continuing through 35 weeks gestation or delivery, whichever occurred first. Women carrying fetus(es) with major congenital anomalies, those with a spontaneous fetal death after 12 weeks, monoamniotic twins, suspected twin-twin transfusion syndrome, marked growth discordance, major uterine anomalies, cerclage or planned cerclage, major chronic medical diseases, and twins resulting from intentional fetal reduction were excluded from randomization in the original trial. All patients had a midtrimester ultrasound to confirm or determine gestational age. The trial found no reduction in PTB $<37$, $<35$, or $<32$ weeks gestation or initial neonatal outcomes with 17-OHPC treatment. The original study was approved by each of the 14 clinical sites' institutional review boards (IRB) and the biostatistics coordinating center. A written informed consent was obtained from all subjects before randomization. This secondary analysis used a de-identified dataset and was considered exempt after review by the IRB at the University of North Carolina, Chapel Hill.

For the purpose of this analysis, only self-identified nonHispanic black and non-Hispanic white women were included. Women of other racial/ethnic groups were excluded due to insufficient sample sizes. Demographic factors (e.g., maternal age, race, educational attainment, marital status), prior pregnancy characteristics (e.g., history of PTB, delivery gestational age of last pregnancy $\geq 14$ weeks), and antenatal factors (e.g., tobacco use, baseline cervical length, bacterial vaginosis, chorionicity, conception by assisted reproductive technology) were compared between non-Hispanic black and non-Hispanic white women. The primary outcome was the gestational age at delivery. Secondary outcomes were selected pregnancy and neonatal outcomes, including a composite of major neonatal morbidity, defined as a diagnosis of at least one of the following complications before initial hospital discharge: fetal or neonatal death, grade III or IV intraventricular hemorrhage, periventricular leukomalacia, bronchopulmonary dysplasia, or necrotizing enterocolitis stage II or III. For neonatal outcomes, the unit of analysis was the pregnancy, and if the outcome occurred in the fetus or neonate, then the pregnancy was considered to have the outcome.

Demographic and clinical characteristics and pregnancy and neonatal outcomes were compared by maternal race using student $t$-test, chi-square, or Fisher's exact test as appropriate. Gestational age at delivery was compared between non-Hispanic black and non-Hispanic white women using Kaplan-Meier survival curves, and the survival distributions were compared using the log-rank test. Cox proportional hazards models estimated hazard ratios (HR) and $95 \%$ confidence intervals (CI) for the association between selected characteristics and delivery gestational age. Stepwise regression with backward elimination was used to select an adjusted model. Initial models included variables significant in bivariate analyses. Main effects were retained in the model if $p$ values were $<0.20$. Variables were considered statistically significant in the final model only if $p$ values were $<0.05$. All statistical analyses were performed with STATA software (version 14.0; Stata Corporation, College Station, TX).

\section{Results}

Of the 661 pregnant women with twin gestations enrolled in the original trial, 535 were self-identified as either nonHispanic black ( $n=150,28.0 \%)$ or non-Hispanic white ( $n=385,72.0 \%$ ) and met inclusion criteria for this secondary analysis. Demographic and clinical characteristics are compared between non-Hispanic black and non-Hispanic white women in - Table 1. Non-Hispanic black women were more likely to be younger, nulliparous, report tobacco use during the current pregnancy, have a history of PTB, preexisting hypertension, bacterial vaginosis, and cervical infection resulting from trichomonas and/or chlamydia during pregnancy as compared with non-Hispanic white women. They were less likely to be married, have a high school education, and conceive via assisted reproductive technology. In addition, non-Hispanic black women had higher body mass index and shorter initial cervical lengths than non-Hispanic white women. Other characteristics were similar between the two groups.

Overall, non-Hispanic black women delivered earlier than non-Hispanic white women (mean delivery gestational age: $33.6 \pm 4.8$ weeks vs. $35.1 \pm 3.1$ weeks; $p<0.001$ ) (data not shown). In addition, a higher proportion of non-Hispanic black women delivered preterm for each of the prespecified gestational age cutoffs: $<28$ weeks ( 13.3 vs. $4.9 \%, p=0.001$ ), $<34$ weeks ( 36.0 vs. $24.7 \%, p=0.009$ ), and $<37$ weeks (77.3 vs. $66.5 \%, p=0.014$ ), - Fig. 1 . 
Table 1 Maternal demographics and clinical characteristics by race

\begin{tabular}{|l|l|l|l|}
\hline Characteristics $^{\text {a }}$ & $\begin{array}{l}\text { Non-Hispanic Black } \\
(\boldsymbol{n}=\mathbf{1 5 0 )}\end{array}$ & $\begin{array}{l}\text { Non-Hispanic White } \\
(\boldsymbol{n}=385)\end{array}$ & $p$ value \\
\hline Maternal age $(\mathrm{y})$ & $26.3 \pm 6.3$ & $31.8 \pm 6.0$ & $<0.001$ \\
\hline Married & $52(34.7)$ & $352(91.4)$ & $<0.001$ \\
\hline Nulliparous & $41(27.3)$ & $199(51.7)$ & $<0.001$ \\
\hline Pre-pregnancy body mass index $\left(\mathrm{kg} / \mathrm{m}^{2}\right)$ & $30.8 \pm 8.3$ & $25.7 \pm 5.8$ & $<0.001$ \\
\hline High school education or greater & $118(78.7)$ & $370(96.1)$ & $<0.001$ \\
\hline Prior preterm birth $<37$ weeks' gestation & $20(14.7)$ & $23(7.4)$ & 0.015 \\
\hline Delivery gestational age of last pregnancy ${ }^{\text {b }}$ & $37.6 \pm 5.3$ & $37.6 \pm 5.0$ & 0.980 \\
\hline Conceived by assisted reproductive technology & $8(5.3)$ & $205(53.3)$ & $<0.001$ \\
\hline Dichorionic placentation & $127(86.4)$ & $333(86.7)$ & 0.922 \\
\hline Bacterial vaginosis & $17(11.3)$ & $3(0.8)$ & $<0.001$ \\
\hline Trichomonas and/or chlamydia & $11(7.3)$ & $5(1.3)$ & 0.001 \\
\hline Baseline transvaginal cervical length obtained & $50(33.3)$ & $124(32.2)$ & 0.803 \\
\hline Initial transvaginal cervical length (mm) & $38 \pm 7$ & $41 \pm 7$ & $1 / 124(0.8)$ \\
\hline Baseline cervical length $<25$ mm & $3 / 50(6.0)$ & $4(1.0)$ & 0.015 \\
\hline Cerclage placed & $5(3.3)$ & 0.072 \\
\hline
\end{tabular}

a Data presented as $n(\%)$ or mean \pm standard deviation.

$b^{b} \geq 14$ weeks among 227 multiparous women (72 black and 155 white).

${ }^{\mathrm{c}}$ After randomization.

Across all gestational ages, the Kaplan-Meier survival analysis demonstrated that non-Hispanic blacks consistently delivered earlier than non-Hispanic whites, log-rank $p=0.004$, -Fig. 2. In the multivariable Cox model, black race (HR: 1.24, 95\% Cl: 1.02-1.51), prior PTB (HR: 1.59, 95\% CI: 1.15-2.19), and the presence of a cervical cerclage (HR: 3.90, 95\% CI: 2.00-7.60) were associated with an increased risk of earlier delivery (-Table $\mathbf{2}$ ).

Pregnancy and neonatal outcomes are compared by race in - Table 3. Non-Hispanic black women were more likely to have spontaneous deliveries (due to spontaneous onset of contractions or rupture of membranes leading to delivery) compared with non-Hispanic whites (66.7 vs. 50.4\%, $p=0.001$ ). Among those delivering due to medical or fetal indications, nonHispanic black women were more likely to deliver due to fetal complications including oligohydramnios, nonreassuring fetal status, and intrauterine growth restriction. Non-Hispanic blacks were less likely to deliver live births (95.3 vs. $98.7 \%$, $p=0.018)$. Fetal or neonatal death (9.3 vs. $3.1 \% ; p=0.006)$ and composite major neonatal morbidity (12.7 vs. $7.0 \%$, $p=0.036)$ rates were higher among non-Hispanic blacks compared with non-Hispanic whites. In limited regression models, both gestational age and black race remained significant modifiers of the risk for the major composite neonatal morbidity ( $p<0.20$, data not shown). Additional outcomes are shown in $\mathbf{- T a b l e ~} 3$.

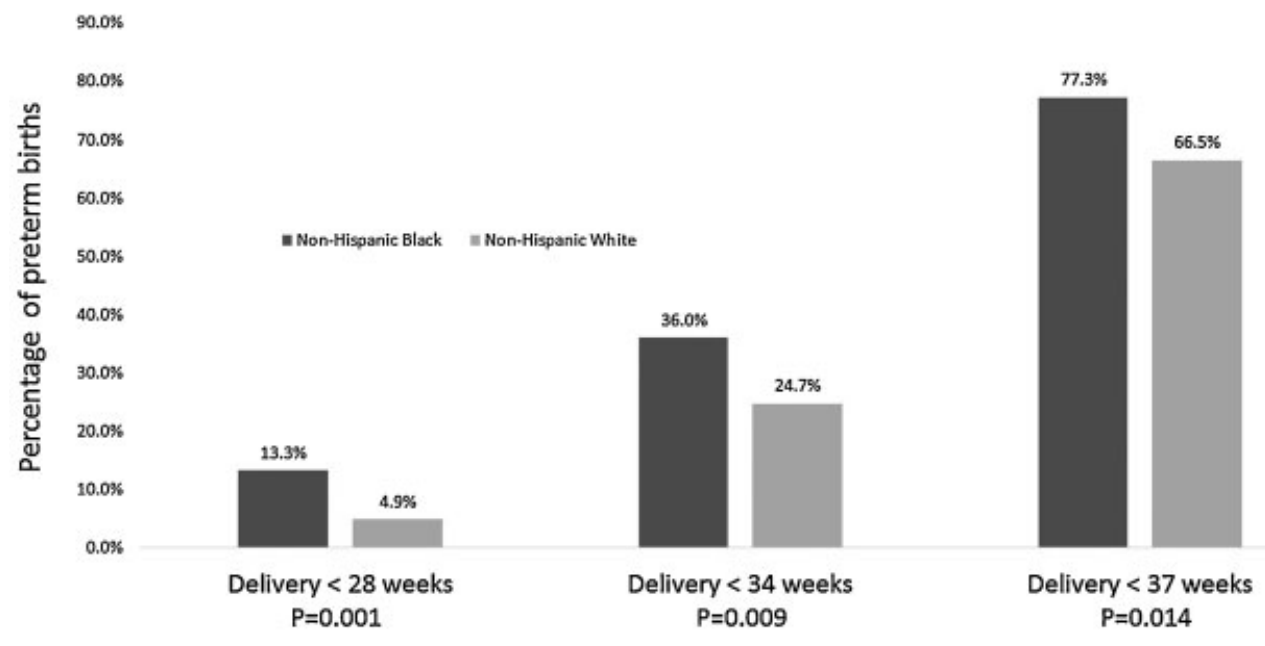

Fig. 1 Percentage of preterm births by maternal race. 


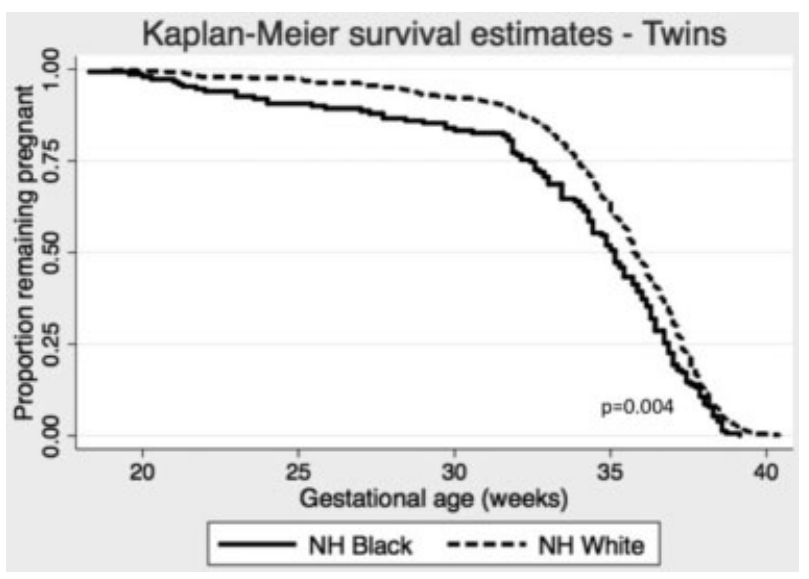

Fig. 2 Kaplan-Meier survival curves of proportions of twin gestations remaining pregnant, by maternal race.
Table 2 Multivariable Cox regression of factors related to gestational age at delivery in twin gestations

\begin{tabular}{|l|l|l|l|}
\hline Characteristics & $\begin{array}{l}\text { Hazard } \\
\text { ratio }^{\mathbf{a}}\end{array}$ & $\begin{array}{l}\text { 95\% Confidence } \\
\text { interval }\end{array}$ & p Value \\
\hline Non-Hispanic black & 1.24 & $1.02-1.51$ & 0.031 \\
\hline Prior preterm birth & 1.59 & $1.15-2.19$ & 0.004 \\
\hline Cerclage & 3.90 & $2.00-7.60$ & $<0.001$ \\
\hline Tobacco use & 1.22 & $0.93-1.60$ & 0.142 \\
\hline
\end{tabular}

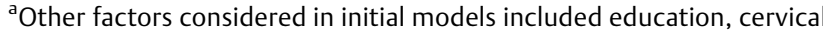
infection with trichomonas or chlamydia, and history of one or more preterm births.

Table 3 Pregnancy and neonatal outcomes

\begin{tabular}{|c|c|c|c|}
\hline Outcomes $^{a}$ & $\begin{array}{l}\text { Non-Hispanic black } \\
(n=150)\end{array}$ & $\begin{array}{l}\text { Non-Hispanic white } \\
(n=385)\end{array}$ & $\mathrm{p}$ value \\
\hline Preeclampsia or gestational hypertension & $25(16.7)$ & $74(19.2)$ & 0.494 \\
\hline Oligohydramnios & $4(2.7)$ & $12(3.1)$ & $>0.99$ \\
\hline Polyhydramnios & $1(0.7)$ & $4(1.0)$ & $>0.99$ \\
\hline Abruption & $5(3.3)$ & $7(1.8)$ & 0.332 \\
\hline Chorioamnionitis & $3(2.0)$ & $6(1.6)$ & 0.716 \\
\hline Preterm premature rupture of membranes & 19 (12.9) & $46(12.1)$ & 0.797 \\
\hline Received tocolysis at any time & $40(26.9)$ & $109(28.3)$ & 0.735 \\
\hline Received antenatal corticosteroids & $41(27.5)$ & $102(26.5)$ & 0.811 \\
\hline Delivered due to spontaneous labor or rupture of membranes & $100(66.7)$ & $194(50.4)$ & 0.001 \\
\hline Delivered due to maternal and/or fetal indications: & $50(33.3)$ & $191(49.6)$ & 0.001 \\
\hline Hypertensive disorders of pregnancy & $14 / 50(28.0)$ & $64 / 189(33.9)^{\mathrm{b}}$ & 0.432 \\
\hline $\begin{array}{l}\text { Nonreassuring fetal status, fetal growth restriction, } \\
\text { oligohydramnios }\end{array}$ & $17 / 50(34.0)$ & $35 / 189(18.5)$ & 0.018 \\
\hline Vaginal delivery & $60(40.5)$ & $161(42.2)$ & 0.737 \\
\hline Livebirth (both twins) & $141 / 148(95.3)$ & $377 / 382(98.7)$ & 0.018 \\
\hline Birthweight (first-born twin) & $2085.8 \pm 602.0$ & $2363.9 \pm 630.2$ & 0.001 \\
\hline Delivered between enrollment and 23 weeks gestation & $9(6.0)$ & $8(2.1)$ & 0.028 \\
\hline Neonatal intensive care unit admission & $70(49.7)$ & $177(47.0)$ & 0.585 \\
\hline Neonatal sepsis & $7(5.0)$ & $12(3.2)$ & 0.337 \\
\hline Respiratory distress syndrome & $22(15.6)$ & $40(10.6)$ & 0.119 \\
\hline Composite major neonatal morbidity & $19(12.7)$ & $27(7.0)$ & 0.036 \\
\hline Necrotizing enterocolitis, stage II or III & $1(0.7)$ & $1(0.3)$ & 0.471 \\
\hline Intraventricular hemorrhage, grade III or IV & $0(0.0)$ & $3(0.8)$ & 0.566 \\
\hline Periventricular leukomalacia & $3(0.80)$ & $2(1.4)$ & 0.617 \\
\hline Bronchopulmonary dysplasia & $3(2.1)$ & $11(2.9)$ & 0.768 \\
\hline Fetal or neonatal death & $14(9.3)$ & $12(3.1)$ & 0.006 \\
\hline
\end{tabular}

${ }^{\text {a } D a t a}$ presented as $n(\%)$ or mean \pm standard deviation.

${ }^{\mathrm{b}}$ Two women with delivery for maternal/fetal indications had unknown specific indications for delivery. 


\section{Comment}

We found that non-Hispanic black women with twin gestations were more likely to deliver at $<37,<34$, and $<28$ weeks. They were also more likely to deliver between study enrollment and 23 weeks gestation, though the study design limits our ability to draw conclusions about previable delivery in this population. On average, non-Hispanic blacks delivered 1.5 weeks earlier than non-Hispanic whites. In our adjusted model, black race, history of PTB, and cerclage presence were associated with earlier delivery. We also found that non-Hispanic blacks were less likely to deliver live births. The rates of composite major neonatal morbidity and perinatal death were higher for non-Hispanic blacks compared with non-Hispanic whites.

Previous studies have examined racial disparities in PTB, but most have been limited to study populations of singleton gestations. ${ }^{11,12,16-20}$ These studies have consistently demonstrated that non-Hispanic black women deliver earlier than non-Hispanic white women. Our study is one of only a few examining the association between black race and gestational age at delivery in twin gestations. Using U.S. birth records from 1990 to 1995, Rolett and Kiely found that non-Hispanic black women with twin gestations had a $30 \%$ higher risk of PTB $<35$ weeks than non-Hispanic whites. ${ }^{21}$ Only black race and age were associated with PTB among twins in their final model. In a retrospective review of over 9,000 live twin births, Cooperstock et al found that socioeconomic factors, including black race, were associated with PTB, with the association limited predominantly to PTB $<34$ weeks. ${ }^{22}$ They reported a relative risk of 1.61 (95\% CI: $1.46,1.76 ; p<0.001)$ for PTB $<34$ weeks comparing blacks with whites. In a large retrospective review of pregnancies resulting from in vitro fertilization (IVF), Xiong et al found that non-Hispanic blacks with twin gestations had higher adjusted odds of delivering preterm at $<28$ weeks (odds ratio [OR]: 3.3 ; 95\% CI: $2.7,3.9$ ), $<32$ weeks (OR: 2.4; 95\% CI: 2.1, 2.7), and $<37$ weeks (OR: $1.4 ; 95 \% \mathrm{CI}: 1.2,1.6$ ) as compared with non-Hispanic whites. ${ }^{23}$ Their analysis, which also reviewed singleton IVF pregnancies, revealed similar patterns of racial differences among IVF twin and singleton gestations.

The association of race and PTB has been described less consistently in twin gestations. To et al found that only cervical length was predictive of PTB $<32$ weeks in twin gestations and the addition of maternal characteristics, such as race, did not improve predictability as it had in singleton gestations. ${ }^{24,25}$ Likewise, black race was not associated with PTB at $<32,<35$, and $<37$ weeks in a prospective study of 150 women with twin gestations. ${ }^{26}$ Our sample size was nearly four times larger than their sample size, which had 50 white patients, and was sufficiently powered to demonstrate the association of race and PTB.

The strong association we observed between the history of PTB and gestational age at delivery for recurrent twin gestations was expected. The association between prior PTB and recurrent PTB has been reported in singleton and twin gestations. ${ }^{27-29}$ Our findings pertaining to cerclage use in twins were not unanticipated. Patients with a history of indicated or planned cerclages were excluded before randomization in the original study. Therefore, patients treated with cerclage in our analysis had either examination or ultrasoundindicated cerclages and were inherently at higher risk of preterm delivery. Nonetheless, the utility of cerclage in twins remains questionable. ${ }^{30}$

While many previous studies investigating the relationship between race and twin PTB demonstrated that black women had higher odds of delivering preterm, these studies were retrospective analyses and did not explore the effect of race on PTB throughout gestation or on neonatal outcomes. Historically, black race was thought to confer protection to preterm neonates such that black preterm neonates had reduced risks of death and serious neonatal morbidity. ${ }^{31}$ However, this traditional paradigm was recently challenged by findings from a large retrospective review of 19,325 singleton preterm deliveries that demonstrated an increased likelihood of intraventricular hemorrhage, intracranial hemorrhage, and perinatal death among black preterm neonates. ${ }^{32}$ Our finding that neonates born to black mothers had increased rates of morbidity is similar to the findings from this prior study. Unfortunately, though both were important in limited regression models, we were underpowered to fully evaluate the contribution of race compared with gestational age to major neonatal morbidity.

Our study has several strengths. It is a secondary analysis of a large multicenter prospective randomized trial. Trained data abstractors utilized a consistent data extraction tool. Gestational age is well-validated, with all patients undergoing a midtrimester ultrasound.

Our study also has limitations. Black and white race was self-identified, and we did not have any data on country of origin or birthplace. However, this is a multicenter study including participants with varied backgrounds. We included all PTBs and made no distinction between spontaneous and indicated PTB for the primary and secondary analysis. However, the presumed effect of indicated PTBs was minimized by excluding monoamniotic twins, those with suspected twin-twin transfusion syndrome, growth discordance, severe fetal anomalies, a spontaneous fetal death after 12 weeks and pregnancies complicated by major maternal chronic diseases. Resultant neonatal outcomes thus were primarily dependent on gestational age at delivery regardless of whether they were indicated or spontaneous. However, the sample size was insufficient to conduct multivariate analyses for pregnancy and neonatal outcomes. Thus there is the potential for residual confounding. Fetal fibronectin and urine drug screens were not conducted for all patients, thus precluding the ability to test the association between PTB and positive fetal fibronectin and urine drug screens.

In summary, our results demonstrate that non-Hispanic black women with twin gestations are at an increased risk of delivering their babies earlier than non-Hispanic white women. Their infants are also more likely to suffer from major morbidity or mortality. These findings parallel 
previously reported PTB disparities in singleton gestations. While interventions to decrease PTB in twin gestations have been largely unsuccessful compared with singleton gestations, these findings warrant measures to increase provider awareness for better risk communication. Hypervigilance is also needed to ensure timely evaluation of these patients who are not only at an increased risk of PTB but whose offspring are not protected from the ill effects of prematurity. Providers should educate non-Hispanic black patients with singleton as well as twin gestations on the signs and symptoms of preterm labor early and often. Additionally, these patients should be informed of the value of timely assessments to implement proven time-sensitive interventions associated with improved neonatal outcomes such as neuroprotective magnesium and antenatal steroids. Future studies are needed to determine protective and effective riskmitigating factors in non-Hispanic blacks with singleton as well as multifetal pregnancies.

\section{Condensation}

Non-Hispanic black women with twin gestations deliver earlier than non-Hispanic white women.

\section{Note}

This article was presented as a poster at the 2017 Annual Meeting of the Society for Maternal-Fetal Medicine; January 26, 2017; Las Vegas, NV. Final abstract ID\# 149.

\section{Funding}

None.

\section{Conflict of Interest}

None.

\section{References}

1 Matthews TJ, MacDorman MF, Thoma ME. Infant mortality statistics from the 2013 period linked birth/infant death data set. Natl Vital Stat Rep 2015;64(09):1-30

2 Sutton PS, Darmstadt GL. Preterm birth and neurodevelopment: a review of outcomes and recommendations for early identification and cost-effective interventions. J Trop Pediatr 2013;59(04): 258-265

3 Hofman PL, Regan F, Jackson WE, et al. Premature birth and later insulin resistance. N Engl J Med 2004;351(21):2179-2186

4 Irving RJR, Belton NR, Elton RA, Walker BR. Adult cardiovascular risk factors in premature babies. Lancet 2000;355(9221): 2135-2136

5 Abitbol CL, Rodriguez MM. The long-term renal and cardiovascular consequences of prematurity. Nat Rev Nephrol 2012;8(05): 265-274

6 Behrman RE, Butler AS; Institute of Medicine (US) Committee on Understanding Premature Birth and Assuring Healthy Outcomes. Preterm Birth: Causes, Consequences, and Prevention. Washington (DC): National Academies Press (US); 2007

7 Martin JA, Hamilton BE, Osterman MJ. Births in the United States, 2015. NCHS Data Brief 2016;258(258):1-8

8 Martin JA, Hamilton BE, Osterman MJ, Driscoll AK, Mathews TJ. Births: final data for 2015. Natl Vital Stat Rep 2017;66(01): $1-70$
9 Goldenberg RL, Culhane JF, Iams JD, Romero R. Epidemiology and causes of preterm birth. Lancet 2008;371(9606):75-84

10 Lu MC, Halfon N. Racial and ethnic disparities in birth outcomes: a life-course perspective. Matern Child Health J 2003;7(01): 13-30

11 Collins JW Jr, David RJ, Simon DM, Prachand NG. Preterm birth among African American and white women with a lifelong residence in high-income Chicago neighborhoods: an exploratory study. Ethn Dis 2007;17(01):113-117

12 Adams MM, Read JA, Rawlings JS, Harlass FB, Sarno AP, Rhodes PH. Preterm delivery among black and white enlisted women in the United States Army. Obstet Gynecol 1993;81(01):65-71

13 Collins JW Jr, Wall SN, David RJ. Adequacy of prenatal care utilization, maternal ethnicity, and infant birthweight in Chicago. J Natl Med Assoc 1997;89(03):198-203

14 Goldenberg RL, Cliver SP, Mulvihill FX, et al. Medical, psychosocial, and behavioral risk factors do not explain the increased risk for low birth weight among black women. Am J Obstet Gynecol 1996; 175(05):1317-1324

15 Rouse DJ, Caritis SN, Peaceman AM, et al; National Institute of Child Health and Human Development Maternal-Fetal Medicine Units Network. A trial of 17 alpha-hydroxyprogesterone caproate to prevent prematurity in twins. N Engl J Med 2007;357(05): 454-461

16 Blackmore-Prince C, Kieke B Jr, Kugaraj KA, et al. Racial differences in the patterns of singleton preterm delivery in the 1988 National Maternal and Infant Health Survey. Matern Child Health J 1999; 3(04):189-197

17 Bediako PT, BeLue R, Hillemeier MM. A comparison of birth outcomes among Black, Hispanic, and Black Hispanic women. J Racial Ethn Health Disparities 2015;2(04):573-582

18 Hibbs S, Rankin KM, David RJ, Collins JW Jr. The relation of neighborhood income to the age-related patterns of preterm birth among White and African-American women: The effect of cigarette smoking. Matern Child Health J 2016;20(07):14321440

19 Kramer MR, Hogue CR. Place matters: variation in the black/ white very preterm birth rate across U.S. metropolitan areas, 2002-2004. Public Health Rep 2008;123(05):576-585

20 McKinnon B, Yang S, Kramer MS, Bushnik T, Sheppard AJ, Kaufman JS. Comparison of black-white disparities in preterm birth between Canada and the United States. CMAJ 2016;188(01): E19-E26

21 Rolett A, Kiely JL. Maternal sociodemographic characteristics as risk factors for preterm birth in twins versus singletons. Paediatr Perinat Epidemiol 2000;14(03):211-218

22 Cooperstock MS, Bakewell J, Herman A, Schramm WF. Association of sociodemographic variables with risk for very preterm birth in twins. Obstet Gynecol 1998;92(01):53-56

23 Xiong X, Pridjian G, Dickey RP. Racial and ethnic disparities in preterm births in infants conceived by in vitro fertilization in the United States. Am J Obstet Gynecol 2013;209(02):128.e1128.e6

24 To MS, Skentou CA, Royston P, Yu CK, Nicolaides KH. Prediction of patient-specific risk of early preterm delivery using maternal history and sonographic measurement of cervical length: a population-based prospective study. Ultrasound Obstet Gynecol 2006;27(04):362-367

25 To MS, Fonseca EB, Molina FS, Cacho AM, Nicolaides KH. Maternal characteristics and cervical length in the prediction of spontaneous early preterm delivery in twins. Am J Obstet Gynecol 2006; 194(05):1360-1365

26 Goldenberg RL, Iams JD, Miodovnik M, et al; National Institute of Child Health and Human Development Maternal-Fetal Medicine Units Network. The preterm prediction study: risk factors in twin gestations. Am J Obstet Gynecol 1996;175(4 Pt 1): 1047-1053 
27 Kazemier BM, Buijs PE, Mignini L, Limpens J, de Groot CJ, Mol BW; EBM CONNECT. Impact of obstetric history on the risk of spontaneous preterm birth in singleton and multiple pregnancies: a systematic review. BJOG 2014;121(10):1197-1208, discussion 1209

28 Ananth CV, Kirby RS, Vintzileos AM. Recurrence of preterm birth in twin pregnancies in the presence of a prior singleton preterm birth. J Matern Fetal Neonatal Med 2008;21(05): 289-295

29 Ananth CV, Getahun D, Peltier MR, Salihu HM, Vintzileos AM. Recurrence of spontaneous versus medically indicated preterm birth. Am J Obstet Gynecol 2006;195(03):643-650
30 Jarde A, Lutsiv O, Park CK, et al. Preterm birth prevention in twin pregnancies with progesterone, pessary, or cerclage: a systematic review and meta-analysis. BJOG 2017. Doi: 10.1111/14710528.14513

31 Tyson JE, Parikh NA, Langer J, Green C, Higgins RD; National Institute of Child Health and Human Development Neonatal Research Network. Intensive care for extreme prematuritymoving beyond gestational age. N Engl J Med 2008;358(16): 1672-1681

32 Wallace ME, Mendola P, Kim SS, et al. Racial/ethnic differences in preterm perinatal outcomes. Am J Obstet Gynecol 2017;216(03): 306.e1-306.e12 